CrossMark \& click for updates

Cite this: Mol. BioSyst., 2015, 11,3164

Received 18th June 2015, Accepted 9th September 2015

DOI: $10.1039 / \mathrm{c} 5 \mathrm{mb} 00409 \mathrm{~h}$

www.rsc.org/molecularbiosystems

\title{
Depiction of carbohydrate-active enzyme diversity in Caldicellulosiruptor sp. F32 at the genome level reveals insights into distinct polysaccharide degradation features
}

\author{
Dong-Dong Meng, ${ }^{a}$ Yu Ying, ${ }^{\text {ab }}$ Kun-Di Zhang, ${ }^{a}$ Ming Lu ${ }^{a}$ and Fu-Li Li*a
}

\begin{abstract}
Thermophilic bacterium Caldicellulosiruptor sp. F32 can utilize cellulose-, hemicellulose-containing biomass, including unpretreated wheat straw. We have conducted a bioinformatics analysis of the carbohydrate-active enzyme (CAZyme) in the genome of Caldicellulosiruptor sp. F32, which reveals a broad substrate range of the strain. Among 2285 predicted open reading frames (ORFs), 73 (3.2\%) CAZyme encoding genes, including 44 glycoside hydrolases (GHs) distributing in $22 \mathrm{GH}$ families, 6 carbohydrate esterases (CEs), 3 polysaccharide lyases (PLs), 21 glycosyl transferases (GTs), and 25 carbohydrate-binding modules (CBMs) were found. An in-depth bioinformatics analysis of CAZyme families that target cellulose, hemicellulose, chitin, pectin, starch, and $\beta-1,3-1,4$-glucan degradation were performed to highlight specialized polysaccharide degrading abilities of strain F32. A great number of orthologous multimodular CAZymes of Caldicellulosiruptor sp. F32 were found in other strains of genus Caldicellulosiruptor. While, a portion of the CAZymes of Caldicellulosiruptor sp. F32 showed sequence identity with proteins from strains of genus Clostridium. A thermostable $\beta$-glucosidase BlgA synergistically facilitated the enzymatic degradation of Avicel by endo-1,4- $\beta$-glucanase CelB, which indicated that the synchronous action of synergism between CAZymes enhanced the lignocellulose degradation by Caldicellulosiruptor sp. F32.
\end{abstract}

\section{Introduction}

Thermophilic bacteria of genus Caldicellulosiruptor $\left(T_{\mathrm{opt}}\right.$ $65-78{ }^{\circ} \mathrm{C}$ ) are robust and efficient carbohydrate degrading microorganisms, which secrete a series of carbohydrate active enzymes (CAZymes) to deconstruct lignocellulosic biomass. ${ }^{1}$ Gram-positive Caldicellulosiruptor spp. have been isolated from a wide variety of ecological niches. Most of the species can metabolize extensive monosaccharides, oligosaccharides, and polysaccharides, including crystalline cellulose, xylan, and switchgrass. ${ }^{2}$ Furthermore, since no carbon catabolite repression (CCR) system exists in C. saccharolyticus, this strain has been shown to be able to ferment $\mathrm{C}_{5}$ and $\mathrm{C}_{6}$ sugars simultaneously, which was a highly desirable feature for lignocellulose conversion. ${ }^{3,4}$ All isolates are given a diverse set of cell wall degrading enzymes (CWDEs) as an excellent CAZyme reservoir for carbohydrate

\footnotetext{
${ }^{a}$ Key Laboratory of Biofuels, Shandong Provincial Key Laboratory of Energy Genetics, Qingdao Institute of Bioenergy and Bioprocess Technology, Chinese Academy of Sciences, Qingdao, 266101, China.E-mail: lifl@qibebt.ac.cn; Fax: +86-532-80662778; Tel: +86-532-80662655

${ }^{b}$ Yellow Sea Fisheries Research Institute, Chinese Academy of Fishery Sciences, Qingdao, 266071, China
}

degradation, which are potential consolidated bioprocessing (CBP) platforms for biomass-to-biofuel conversion. Cellulolytic enzyme systems can be divided into two main categories, namely cellulosomal and noncellulosomal. Genus Caldicellulosiruptor adopts an intermediate strategy, secreting many free multimodular cellulases containing multiple binding and catalytic domains. ${ }^{5}$

Blumer-Schuette et al. have examined the CAZymes of eight numbers of this genus. Each strain contains a significant number of glycoside hydrolases (GHs, 37-77), carbohydrate binding modules (CBMs, 15-28), polysaccharide lyases (PLs, 1-4), carbohydrate esterases (CEs, 4-9), and glycosyl transferases (GTs, 27-35), ${ }^{2}$ which comprise 1-3\% of the genome. Comparison analysis within the genus from the genome level indicates that $106 \mathrm{GHs}$ distributing in $43 \mathrm{GH}$ families constitute the Caldicellulosiruptor pangenome, while $26 \mathrm{GHs}$ from 17 families are conserved in the core genome. ${ }^{2}$ Though more than half of the lignocellulose degradation related proteins are shared between all Caldicellulosiruptor species, genomic and proteomic studies in one individual species are needed to distinguish the difference of carbohydrate utilization within the genus. ${ }^{6}$ As an efficient lignocellulolytic bacterium toward unpretreated plant biomass, the analysis of CAZyme families 
and their architecture composition at the genome level is helpful to elucidate the ability of Caldicellulosiruptor species for the deconstruction of plant polysaccharides.

Caldicellulosiruptor sp. F32 is an extremely thermophilic anaerobic bacterium, which was isolated from biocompost with optimal growth temperature at $75{ }^{\circ} \mathrm{C}{ }^{7}$ Compared with the type stain C. saccharolyticus DSM 8903, Caldicellulosiruptor sp. F32 grew better on unpretreated wheat straw, exhibiting higher endoglucanase and xylanase activities in the secretome. ${ }^{8}$ Three CAZy enzymes, GH11 xylanase XynA (GenBank accession number JX030400), GH10 xylanase XynB (GenBank accession number JX030401), ${ }^{9}$ and GH5 lichenase F32EG5 (GenBank accession number KC958563, unpublished), have been characterized in detail. Further elucidation of the abundant CAZymes is thus essential to understand the complexity process of the lignocellulose deconstruction. This report focused on the CAZyme identification of strain F32 and provided a wealth of information to generate a comprehensive analysis of CWDEs in the host. Sequence annotation and module annotation have been applied to predict functions of each CAZyme protein. Moreover, synergism between two cellulose-degrading enzymes was investigated. Briefly, this will provide a better understanding of the plant-biomass degradation mechanisms of the genus Caldicellulosiruptor.

\section{Materials and methods}

\section{Genomic DNA isolation and genome sequencing}

Caldicellulosiruptor sp. F32 (CGMCC 1.5183, China General Microbiological Collection Center, Beijing, China) was cultured at $75{ }^{\circ} \mathrm{C}$ in an anaerobic jar containing the modified GS-2 medium. ${ }^{7}$ Genomic DNA was isolated ${ }^{10}$ from Caldicellulosiruptor sp. F32 and was sequenced by a standard shotgun strategy using GS FLX-454 pyrosequencing technology (Roche, Basel, Switzerland). The numerous reads were assembled by using the 454 Newbler assembler software (Roche) into hundreds of contigs, which were re-sorted subsequently to predict gene functions using Glimmer, ${ }^{11}$ GeneMark, ${ }^{12}$ and Zcurve. ${ }^{13}$ The genes were annotated through the National Center for the Biotechnology Information (NCBI) $n r$ database ${ }^{14}$ and the KEGG database. ${ }^{15}$

\section{CAZyme annotation}

All protein-encoding open reading frames (ORFs) from the Caldicellulosiruptor sp. F32 genome generated through $\mathrm{nr}$ and KEGG database annotation were further refined and screened for carbohydrate-active modules using Carbohydrate-active enzyme ANnotatio (dbCAN, http://csbl.bmb.uga.edu/dbCAN/ index.php). ${ }^{16}$ To eliminate proteins identified by dbCAN but those were not really CAZymes, all proteins were individually examined using the NCBI's Conserved Domain Database (CDD) (http://www.ncbi.nlm.nih.gov/Structure/cdd/wrpsb.cgi) ${ }^{17}$ and Pfam (http://pfam.sanger.ac.uk/). ${ }^{18}$ These data sources were combined to assert the function of each predicted proteins. When a gene appeared to be truncated and the missing sequence data could not be found within all the supercontig, the orthologous coding region in other Caldicellulosiruptor isolates was used to modify the gene sequence from Caldicellulosiruptor sp. F32 genome. Further annotation of selected proteins included the determination of protein similarities and identities, ${ }^{19}$ molecular size and isoelectric point prediction, ${ }^{20}$ and signal peptide prediction (SignalP v4.1). ${ }^{21}$

\section{Cloning, expression, and purification of Caldicellulosiruptor sp. F32 GHs}

The primers BlgA-F 5'-ATGAGTTTCCCAAAAGGATTTTTG-3' and BlgA-R 5'-GTATATAAAGGAAAATTCGTAA- $3^{\prime}$ were used to amplify the $\beta$-glucosidase (BlgA) encoding gene. The primers CelB-F $5^{\prime}$-CAAAATACTGCGTATGAAAAGG- ${ }^{\prime}{ }^{\prime}$ and CelB-R $5^{\prime}$-TTACATC TTTCCTGTAAGTTCTAAAATTTTG- $3^{\prime}$ were used to amplify the coding gene of endo- $\beta$-glucanase (CelB) without $\mathrm{N}$-terminal signal peptides (1-28). The PCR product was cloned into the His tag expression vector pEASY-E1 (TransGen, Beijing, China) and transformed into Escherichia coli BL21 (DE3). The nucleotide sequence was verified via sequencing by GenScript (Nanjing, China). Protein expression was induced by using $1 \mathrm{mM}$ isopropyl$\beta$-D-thiogalactopyranoside (IPTG). Cells were harvested $16 \mathrm{~h}$ after shaking at $16{ }^{\circ} \mathrm{C}$ and resuspended in lysis buffer $\left(50 \mathrm{mM} \mathrm{NaH}_{2} \mathrm{PO}_{4}\right.$, $300 \mathrm{mM} \mathrm{NaCl}, \mathrm{pH}$ 8.0) containing protease inhibitor cocktail (Amresco, Solon, USA). The resuspended cells were disrupted using ultrasonication on ice. Then, the crude enzyme was heated at $70{ }^{\circ} \mathrm{C}$ for $10 \mathrm{~min}$ and centrifuged at $4{ }^{\circ} \mathrm{C}$ to remove denaturated $E$. coli proteins. The enzyme solution was applied to a Ni-NTA-Sefinose column (Sangon, Shanghai, China). The active fraction was concentrated and replaced with buffer by ultrafiltration using a $10 \mathrm{kDa}$ cutoff membrane (Millipore, Billerica, MA) at $4{ }^{\circ} \mathrm{C}$. Finally, the purified recombinant proteins were analyzed by using SDS-PAGE. Protein concentration was measured using the Bradford method with bovine serum albumin as the standard. ${ }^{22}$

\section{Biochemical characterization of the recombinant enzymes}

Two buffers, acetate buffer (0.2 M, pH 4.0-5.6) and PC buffer (50 mM phosphate, $12 \mathrm{mM}$ citrate, $\mathrm{pH}$ 6.0-8.0), were used for $\mathrm{pH}$ profiling. For the determination of optimal temperature, the enzymatic reaction was carried out at different temperatures ranging from 65 to $85{ }^{\circ} \mathrm{C}$ with a $5{ }^{\circ} \mathrm{C}$ interval at $\mathrm{pH}$ 5.6. The half-life $\left(t_{1 / 2}\right)$ was obtained by measuring the residual activity of the enzyme at $75{ }^{\circ} \mathrm{C}$ and $\mathrm{pH}$ 5.6. The specific activity of the purified recombinant using $1 \%(\mathrm{~W} / \mathrm{V})$ carboxymethylcellulose (CMC), Avicel PH-101, and cellobiose as substrates was determined. The reducing sugars released were measured using the dinitrosalicylic acid (DNS) method by reading the absorbance at a wavelength of $540 \mathrm{~nm}$ with glucose used as a standard. $p$-Nitrophenyl- $\beta$-D-cellobioside ( $p$ NPC) was used at a final concentration of $1 \mathrm{mM}$ to determine the substrate-specific activity of the enzymes. $p$-Nitrophenol was measured by reading the absorbance at $405 \mathrm{~nm}$ to calculate activity. All cellulosic substrates were purchased from Sigma-Aldrich (St Louis, MO, USA). One unit (U) of enzyme activity was defined as the amount of enzyme that liberated $1 \mu \mathrm{mol}$ of reducing sugar or $p$-nitrophenol per min. Unless otherwise stated, the reaction was performed at triplicate.

Each cello-oligosaccharide (cellobiose, cellotriose, cellotetraose, cellopentaose, and cellohexaose) at a final concentration 
of $10 \mathrm{mg} \mathrm{mL}{ }^{-1}$ was incubated with $0.5 \mu \mathrm{g}$ BlgA in a total volume of $10 \mu \mathrm{L}$ for $12 \mathrm{~h}$ at $75{ }^{\circ} \mathrm{C}$, respectively. The hydrolysis products were analyzed using the thin-layer chromatography (TLC) method by using silica gel plates 60F254 (Merck, Darmstadt, Germany). The plates were developed with a butan-1-ol-acetic acid-water $(2: 1: 1$, $\mathrm{v} / \mathrm{v}$ ) solvent system. The color reaction was carried out in an oven at $105{ }^{\circ} \mathrm{C}$ for $30 \mathrm{~min}$ after spraying the plates with a methanolsulfuric acid mixture $(4: 1, \mathrm{v} / \mathrm{v})$. Oligosaccharides were purchased from Megazyme (Wicklow, Ireland) and used as the standard. The $p$ NPCase of BlgA was performed with various glucose in a final concentration of $0-1000 \mathrm{mM}$ at $75{ }^{\circ} \mathrm{C}$ and $\mathrm{pH} 5.6$ to determinate the glucose-tolerance of BlgA.

Time course hydrolysis of Avicel by CelB was performed at a volume of $1 \mathrm{~mL}$ containing a portion $(50 \mathrm{mg})$ of Avicel and $10 \mu \mathrm{g}$ CelB at $75{ }^{\circ} \mathrm{C}$ and $\mathrm{pH}$ 5.6. At different time intervals $(0 \mathrm{~h}$, $1 \mathrm{~h}, 3 \mathrm{~h}, 6 \mathrm{~h}, 12 \mathrm{~h}, 24 \mathrm{~h}$, and $36 \mathrm{~h}$ ), aliquots were taken and subjected to high-performance anion exchange chromatography with pulsed amperometric detection (HPAEC-PAD, Dionex Corporation, Sunnyvale, CA, USA) analysis to detect end products as described. ${ }^{23}$ A synergy experiment was performed in triplicate in a $1 \mathrm{~mL}$ reaction mixture containing $50 \mathrm{mg}$ Avicel and $10 \mu \mathrm{g}$ CelB with the supplementation dosage of BlgA at $0.1 \mu \mathrm{g}, 0.5 \mu \mathrm{g}$, and $2 \mu \mathrm{g}$, respectively. The reaction was performed at $75{ }^{\circ} \mathrm{C}$ and $\mathrm{pH} 5.6$ for $36 \mathrm{~h}$, and reducing sugars were detected by the DNS method. The degree of synergy (DOS) was calculated as reported before. ${ }^{24}$

\section{Nucleotide sequence accession numbers}

The Caldicellulosiruptor sp. F32 genome sequence and annotation data have been deposited in GenBank under accession number APGP00000000. The GenBank accession numbers for the coding sequences of $\beta$-glucosidase BlgA and endo- $\beta$-glucanase CelB are JX030398 and JX030399, respectively.

\section{Results and discussion}

\section{Identification of CAZyme modules in Caldicellulosiruptor sp. F32}

A total of 127 contigs constituting 24 scaffolds were generated, yielding a total sequence of 2378643 bp of the Caldicellulosiruptor sp. F32 draft genomic DNA, which is shorter than the genome of type strain C. saccharolyticus DSM 8903 (2970275 bp). The protein coding genes of Caldicellulosiruptor sp. F32 were 2285, which account for $85.3 \%$ ORFs of C. saccharolyticus DSM 8903. The genome of Caldicellulosiruptor sp. F32 has a G + C content of $35.2 \%$, and no plasmid was found. Our analysis indicated that $3.2 \%$ (73 of 2285) predicted proteins encoded in the genome of Caldicellulosiruptor sp. F32 are CAZymes involved in lignocellulosic biomass deconstruction. This value is similar to the numbers of CAZymes found in most genus of Caldicellulosiruptor. ${ }^{2}$ A total of 44 ORFs containing one or two GHs have been found in the genome of Caldicellulosiruptor sp. F32, while in the type strain C. saccharolyticus DSM 8903 there are 59 ORFs containing the GH domain. We noticed that the number of GHs in strain F32 is greater than that in C. kristjanssonii (37). Other than GH containing ORFs, the carbohydrate-binding modules (23), polysaccharide
Table 1 Analysis of ORFs from Caldicellulosiruptor sp. F32

\begin{tabular}{lll}
\hline & $\begin{array}{l}\text { Caldicellulosiruptor } \\
\text { Sp. F32 }\end{array}$ & $\begin{array}{l}\text { C. saccharolyticus } \\
\text { DSM 8903 ref. 2 }\end{array}$ \\
\hline Total ORFs of strain & 2285 & 2679 \\
Hypothetical protein & 358 & 781 \\
Amylase & 8 & 4 \\
S-layer domain-containing & 7 & 8 \\
protein & & \\
ABC transporters & 22 & 25 \\
& & \\
CAZymes & 44 & 59 \\
Glycoside hydrolase & 3 & 1 \\
Polysaccharide lyase & 6 & 6 \\
Carbohydrate esterase & 21 & 17 \\
Glycosyl transferase & 25 & \\
Carbohydrate binding & & \\
module & &
\end{tabular}

lyases (2), carbohydrate esterases (6), glycosyl transferase (21), and $\mathrm{ABC}$ transporters (22) are also identified in Caldicellulosiruptor sp. F32 (Table 1). In addition, twenty-one glycosyl transferases, distributing in GT families of $2,4,5,28$, and 36 , are identified.

\section{Cellulose deconstruction}

Cellulose formed by $\beta$-1,4-linked glucose residues can be degraded by a synergistic action of three GH enzymes, namely, endo- $\beta-1,4-$ glucanase (EC 3.2.1.4), cellobiohydrolase (EC 3.2.1.91), and $\beta$-glucosidase (EC 3.2.1.21). In addition to GHs, there are auxiliary activity (AA) proteins ${ }^{25}$ and $\mathrm{CBMs}^{26}$ that assist to break the crystal structure of cellulose and act to concentrate enzyme by targeting and proximity effects, respectively. In Caldicellulosiruptor sp. F32, there are three endo- $\beta$-1,4-glucanases, distributing in $\mathrm{GH}$ families of 5, 9, and 48, respectively. Multimodular enzyme CelA (Csac_1076 of C. saccharolyticus or Athe_1867 of $C$. bescii) represented $34.7 \%$ of total secreted proteins in a cellulose based enrichment of $C$. bescii secretome, and it was considered as one of the most important secreted cellulolytic enzymes in the species. ${ }^{27}$ The polypeptide is composed of an N-terminal GH9 and a C-terminal GH48 catalytic domain that are separated by a CBM3c and two CBM3b module, which is also present in Caldicellulosiruptor sp. F32 (Fig. 1). All these two catalytic modules of CelA can synergistically hydrolyze crystalline cellulose, ${ }^{28}$ and can excavate extensive cavities into the surface of the substrate. ${ }^{5}$ In the genome of strain F32, there are two predicted GH5 endo- $\beta$-1,4-glucanases, which may play an important role in cellulose degradation. One GH5 cellulase CelB of strain F32 contains a CBM28 module and three S-layer homology domains showed an amino acid sequence identity of $99 \%$ with Csac_0678 from C. saccharolyticus DSM 8903 (Fig. 1). It has been reported that Csac_0678 is bound to the C. saccharolyticus S-layer and contributed mainly to the hydrolysis of complex polysaccharides and deconstruction of lignocellulosic biomass. ${ }^{29}$ An intracellular GH5 protein, located at 228572 to 229558 (reverse) of Caldicellulosiruptor sp. F32 genome, was predicted as cellulase, which might hydrolyze oligosaccharides that were transferred into cells. In the genome of Caldicellulosiruptor sp. F32, five putative $\beta$-glucosidases belonging to GH1 and GH3 were predicted (Table 2). In Caldicellulosiruptor sp. F32, 25 CBMs 


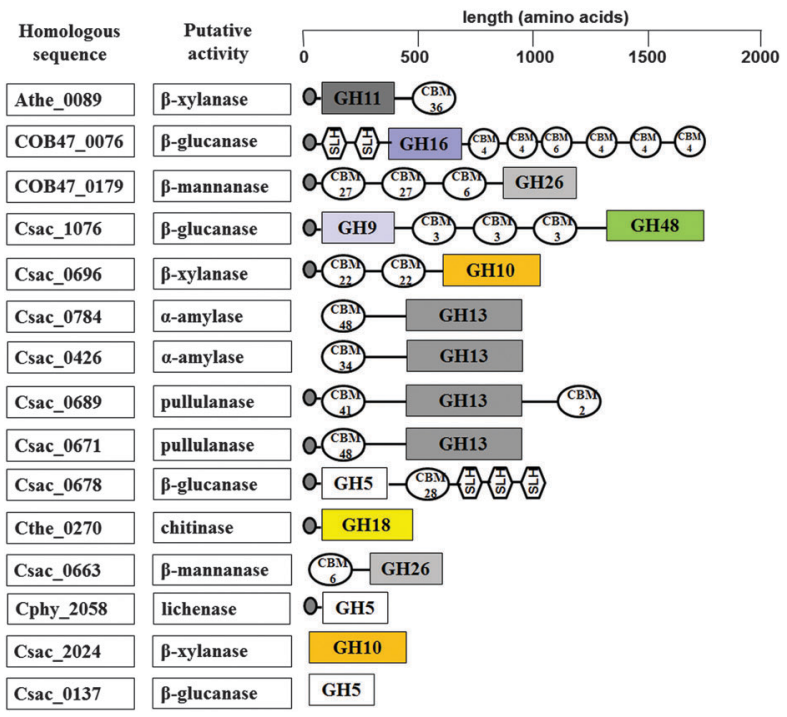

Fig. 1 Glycoside hydrolases (GHs) involved in deconstruction of polysaccharides in Caldicellulosiruptor sp. F32. Csac, C. saccharolyticus; Athe, C. bescii; COB47, C. obsidiansis; Cthe, Clostridium thermocellum; Cphy, Clostridium phytofermentans. The black circle at the $\mathrm{N}$-terminus indicates signal peptide sequence. $\mathrm{GH}$, glycoside hydrolase; CBM, carbohydratebinding module; $\mathrm{SLH}$, surface layer homology domain. Modular arrangement and length of amino acid sequence for each $\mathrm{GH}$ from Caldicellulosiruptor $\mathrm{sp}$. F32 are exhibited on the right of the figure. The putative annotation of each $\mathrm{GH}$ and gene number of its homolog are exhibited on the left of the figure.

distribute in 12 families, and 18 out of these CBMs may be related to cellulose- or glucan-binding. The above analysis suggests that the minimal set of CAZy-related proteins, multimodular GH9-GH48, 2 GH5 enzymes, and $5 \beta$-glucosidases endows Caldicellulosiruptor sp. F32 with the ability to fully degrade cellulose into glucose, which exhibited $0.31 \mathrm{U} \mathrm{mg}^{-1}$ of filter paper activity (FPA), $0.57 \mathrm{U} \mathrm{mg}^{-1}$ of exo-glucanase, and $0.0035 \mathrm{U} \mathrm{mg}^{-1}$ of endo-glucanase in the Avicel induced secreted protein. $^{8}$ This fits well with core genome analysis of eight sequenced genomes of Caldicellulosiruptor. ${ }^{2}$

\section{Hemicellulose degradation}

Hemicellulose, the second most abundant polysaccharides on earth, accounts for $20-35 \%$ of lignocellulosic biomass, ${ }^{30}$ which contains pentose ( $\beta$-D-xylose, $\alpha$-L-arabinose), hexose ( $\beta$-D-mannose, $\beta$-D-glucose, $\alpha$-D-galactose) and/or uronic acid ( $\alpha$-D-glucuronic, $\alpha$-D-4-O-methylgalacturonic and $\alpha$-D-galacturonic acid). ${ }^{31} \mathrm{Hemi}$ cellulose is a heterogeneous group of branched and linear polysaccharides including xyloglucans, xylans, mannans and glucomannans, whose degradation is mediated by several kinds of enzymes. Xylan is the most common hemicellulose and its degradation requires endo-1,4- $\beta$-xylanase (EC 3.2.1.8) and $\beta$-xylosidase (EC 3.2.1.37). In addition, $\alpha$-L-arabinofuranosidase, $\alpha$-glucuronidase, acetylxylan esterase, ferulic acid esterase, and $p$-coumaric acid esterase are necessary for the hydrolysis of various substituted xylans. ${ }^{30}$

Xylanase (endo-1,4- $\beta$-xylanase) hydrolyzes the $\beta-1,4$ bond in the xylan backbone of hemicellulose, yielding short xylooligosaccharides, which are further degraded by $\beta$-xylosidase.
Most xylanases belong to GH families 10 and $11 .{ }^{32}$ Caldicellulosiruptor sp. F32 exhibits distinctive properties in growth and xylan hydrolysis, which was facilitated by the thermostable and efficient set of xylanases in the secreted proteins. ${ }^{7}$ The Caldicellulosiruptor sp. F32 genome contains two multimodular xylanases, namely GH10 XynA and GH11 XynB. ${ }^{9}$ In addition, another ORF encoding GH10 xylanase without signal peptides was found in the genome of strain F32 (Fig. 1) and it might degrade oligosaccharides with a low degree of polymerization. Furthermore, candidate $\beta$-xylosidases including 7 putative $\mathrm{GH} 2$ and 5 putative $\mathrm{GH} 43$ proteins were detected in the genome, and they were annotated as xylan $\beta-1,4$-xylosidase or $\alpha$-Larabinofuranosidase which might hydrolyze oligosaccharides and assist xylanases for the complete degradation of xylan to xylose. Interestingly, homology sequences of these two GH10 xylanases were found in all Caldicellulosiruptor species, while GH11 xylanases were only found in C. bescii (ACM59249.1), C. owensensis (ADQ03663.1), and Caldicellulosiruptor sp. Rt69B.1 (AAB95327.1). It is worth noting that multi-modular extracellular GH11 xylanases possessed 9 fold higher specific activity than GH10 xylanases, and they also showed a degree of synergy of approximately $1.3 .^{9}$ It has been reported that GH11 xylanases are exclusively active on D-xylose containing substrates, while, GH10 xylanases show mainly activity towards xylooligosaccharides and cellulose substrates with low molecular mass. ${ }^{32}$ In the genome of the type strain C. saccharolyticus DSM 8903, six GH10 enzymes encoding ORFs including Csac_1078, Csac_0204, Csac_0696, Csac_2405, Csac_2408, and Csac_2410 were found, but no GH11 xylanase encoding sequence present. Due to the synergistic activity of GH11 and GH10 xylanases found in strain F32, 2.5 fold higher xylanase activity of the secreted proteins in strain F32 has been detected than that in C. saccharolyticus DSM 8903. ${ }^{7}$ As we know, high xylanase activity can lead to improved utilization of lignocellulose during initial and mid-exponential phase of microorganisms, ${ }^{33}$ therefore, we can explain at the genome level now why strain F32 showed better growth $\left(8.3 \times 10^{7}\right.$ cells per $\left.\mathrm{mL}\right)$ than DSM $8903\left(2.5 \times 10^{7}\right.$ cells per $\left.\mathrm{mL}\right)$ when unpretreated wheat straw was used as the sole carbon source. The secreted protein encoded by Csac_2410 is a multi-modular enzyme containing GH5 and GH10 catalytic domains, and activity towards CMC, Avicel, barley $\beta$-glucan, and xylan has been proved. ${ }^{34}$

In Caldicellulosiruptor sp. F32, a panel of glycoside hydrolases including one putative GH4 $\alpha$-galactosidase (EC 3.2.1.22), one putative GH31 $\alpha$-xylosidase (EC 3.2.1.177), five putative GH43 $\alpha$-L-arabinofuranosidases (EC 3.2.1.55), one putative GH30 glucosylceramidase (EC 3.2.1.45), and one putative GH51 $\alpha$-L-arabinofuranosidase (EC 3.2.1.55), which are involved in removing side chains from the xylose backbone of hemicellulose, was identified. In the genome of Caldicellulosiruptor sp. F32, there are 6 putative carbohydrate esterases (acetylxylan esterase, EC 3.1.1.72) belonging to CE4, CE7, and CE9, most of them might shear the O-acetyl groups from positions 2 and/or 3 on the $\beta$-D-xylopyranosyl residues of acetyl xylan, particularly arabinoglucuronoxylan. ${ }^{31}$ In addition to xylan-degrading enzymes, there are 2 putative GH26 $\beta$-mannanases (EC 3.2.1.78) and 7 putative GH2 $\beta$-mannosidases (EC 3.2.1.25) which are related to the degradation of the main chain and 
Table 2 The modules of CAZymes from Caldicellulosiruptor sp. F32

\begin{tabular}{|c|c|c|c|c|}
\hline CAZymes family & Substrate & Enzyme activity & EC number & Number \\
\hline GH1 & Cellulose & $\beta$-Glucosidase & 3.2.1.21 & 1 \\
\hline \multirow[t]{4}{*}{ GH2 } & Hemicellulose & $\beta$-Mannosidase & 3.2.1.25 & 7 \\
\hline & & $\beta$-Glucuronidase & 3.2.1.31 & \\
\hline & & $\beta$-L-Arabinofuranosidase & 3.2.1.55 & \\
\hline & & exo- $\beta$-Glucosaminidase & 3.2.1.165 & \\
\hline & Hemicellulose & Xylan $\beta$-1,4-xylosidase & 3.2.1.37 & \\
\hline & & Glucan $\beta$-1,3-glucosidase & 3.2.1.58 & \\
\hline & & $\alpha$-L-Arabinofuranosidase & 3.2.1.55 & \\
\hline \multirow{2}{*}{ GH4 } & Hemicellulose & $\alpha$-Glucosidase & 3.2.1.20 & 1 \\
\hline & & $\alpha$-Galactosidase & 3.2.1.22 & 1 \\
\hline \multirow[t]{2}{*}{ GH5 } & Cellulose & Cellulase & 3.2.1.4 & 2 \\
\hline & & Lichenase & 3.2.1.73 & 1 \\
\hline & Pullulan & Pullulanase & 3.2.1.41 & 2 \\
\hline GH15 & Starch & Glucoamylase & 3.2.1.3 & 1 \\
\hline \multirow[t]{2}{*}{ GH16 } & Hemicellulose & endo- $\beta-1,4$-Glucanase & 3.2 .1 .151 & 1 \\
\hline & $\beta$-1,3-Glucans & Glucan endo- $\beta$-1,3-glucosidase & 3.2.1.39 & \\
\hline \multirow[t]{2}{*}{ GH18 } & Chitin & Chitinase & 3.2.1.14 & 1 \\
\hline & & endo- $\beta$ - $N$-Acetylglucosaminidase & 3.2.1.96 & \\
\hline GH20 & Hemicellulose & $\mathrm{N}$-Acetylglucosaminidase & 3.2 .1 .30 & 1 \\
\hline GH26 & Mannan & $\beta$-Mannanase & 3.2.1.78 & 2 \\
\hline GH29 & Hemicellulose & $\alpha$-L-Fucosidase & 3.2.1.51 & 1 \\
\hline GH30 & Hemicellulose & Glucosylceramidase & 3.2.1.45 & 1 \\
\hline \multirow[t]{2}{*}{ GH31 } & Starch & $\alpha$-Glucosidase & 3.2.1.20 & 1 \\
\hline & Hemicellulose & $\alpha$-Xylosidase & 3.2.1.177 & 1 \\
\hline \multirow{2}{*}{$\begin{array}{l}\text { GH42 } \\
\text { GH43 }\end{array}$} & Hemicellulose & $\beta$-Galactosidase & 3.2.1.23 & 1 \\
\hline & Hemicellulose & $\alpha$-L-Arabinofuranosidase & 3.2.1.55 & 5 \\
\hline CE7 & Hemicellulose & Acetyl xylan esterase & 3.1.1.72 & 1 \\
\hline CE9 & Hemicellulose & $\mathrm{N}$-Acetylglucosamine 6-phosphate deacetylase & 3.5.1.25 & 1 \\
\hline \multicolumn{5}{|c|}{ Polysaccharide lyase (PL) } \\
\hline PL3 & Pectin (HG, RGI) & Pectate lyase & 4.2.2.2 & 2 \\
\hline PL11 & Pectin & Rhamnogalacturonan lyase & 4.2 .2 & 1 \\
\hline \multicolumn{5}{|c|}{ Carbohydrate binding module (CBM) } \\
\hline CBM2 & Cellulose, chitin, xylan & Cellulose-binding & & 1 \\
\hline CBM3 & Cellulose, chitin & Cellulose-binding & & 4 \\
\hline CBM4 & Glucan & Glucan-binding & & 5 \\
\hline CBM6 & Glucan & Glucan-binding & & 3 \\
\hline CBM22 & Xylan & Xylan-binding & & 3 \\
\hline CBM27 & Mannan & Mannan-binding & & 2 \\
\hline CBM28 & Glucan & Cellulose-binding & & 1 \\
\hline CBM34 & Starch & Starch-binding & & 1 \\
\hline CBM36 & Xylan & Xylan-binding & & 1 \\
\hline CBM41 & Pullulan & Glucan-binding & & 1 \\
\hline CBM48 & Glycogen & Glycogen-binding & & 2 \\
\hline CBM50 & Peptidoglycan & Peptidoglycan-binding & & 1 \\
\hline
\end{tabular}

side chains of glucomannans. CBMs are usually considered as the auxiliary domain in conjunction with other CAZyme catalytic modules. ${ }^{35}$ The traditional recognized function of non-catalytic CBMs is to bind polysaccharides, bringing the biocatalyst into its substrates by targeting and proximity effects. ${ }^{26}$ There are over 70 families of CBMs, and these CBMs are classified into three types based on their binding preference towards crystalline polysaccharides, soluble polysaccharides, 
and soluble mono-, di- or tri-saccharides, respectively. ${ }^{36}$ It is illusory that the deletion of CBMs from the multi-modular GH10 and GH11 xylanases from Caldicellulosiruptor sp. F32 resulted in an opposite effect on thermostability of the truncated mutants. Through homology modeling and cross-linking analysis, it turned out to be that the optimized intramolecular interaction between CBM and the catalytic domain conveyed improved thermostability of GH10 xylanase. ${ }^{9}$

\section{Chitin degradation}

Chitin, a linear polymer of $\beta-1,4-N$-acetylglucosamine (GlcNAC), is found in the outer skeleton of insects, fungi, yeasts, algae, crabs, shrimps, and lobsters, and in the internal structure of other invertebrates. ${ }^{37}$ Chitinases (E.C 3.2.1.14) are a widespread group of glycosyl hydrolases found in bacteria, fungi, yeasts, plants, actinomycetes, and arthropods. ${ }^{38}$ The enzymes act on the $N$-acetyl- $\beta-1,4$-glucosaminide linkages in chitin and randomly split chitin at internal sites to produce oligosaccharides such as chitotriose, and chitotetraose. ${ }^{39}$ Caldicellulosiruptor sp. F32 contains a putative extracellular GH18 chitinase (Table 2), which shares the highest amino acid identity of $40 \%$ with chitinase LpChiA from Laceyella putida (BAO37115.1). ${ }^{40}$ Members from the GH18 family usually show chitinase or endo- $N$-acetyl- $\beta$-1,4-glucosaminidase activity. ${ }^{41}$ In the genome of Caldicellulosiruptor sp. F32, a GH20 putative $N$-acetylglucosaminidase (EC 3.2.1.30) was predicated, which might play a role in the cleavage of oligomeric products of chitinase, thereby generating monomers of GlcNAc. ${ }^{39}$

\section{Pectin degradation}

Pectin is mainly constructed by three types of polysaccharides including homogalacturonan (HG, 1,4-linked $\alpha$-D-galactopyranosyluronic acid, GalpA), rhamnogalacturonan-I (RG-I, alternative $\alpha$-1,2-rhamnosyl and $\alpha$-1,4-galacturonic acid), and RG-II ( $\alpha$-1,4linked GalpA). ${ }^{42,43}$ A panel of CAZymes, distributing in GHs, CEs, and PLs, is required for complete deconstruction of the complex pectin. Usually, GH28 endopolygalacturonases (EC 3.2.1.15) and exopolygalacturonases (EC 3.2.1.67) can cleave $\alpha$-1,4-linkages and $\alpha-1,2$-linkages in pectin, respectively. To date, CAZymes from the GH1, GH2, GH3, GH5, GH30, GH35, GH43, GH53, and GH54 families have been reported to be able to degrade side chains of pectin. ${ }^{43}$ In the genome of Caldicellulosiruptor sp. F32, though GH28 CAZyme was not found, a GH105 putative rhamnogalacturonyl hydrolase (EC 3.2.1.172) was considered to be involved in the cleavage of rhamnogalacturonan. A homology of Cbes_1853, which has been proposed as rhamnogalacturonan lyase in $C$. bescii, containing the modules of PL11 and CBM3, is also present in the genome of strain F32. ${ }^{44}$ Furthermore, two PL3 CAZymes annotated as pectate lyase in Caldicellulosiruptor sp. F32 might cleave bonds linking the $\alpha$-1,4-galacturonan residues to the HG backbone at the non-reducing end. ${ }^{43}$

\section{Starch degradation}

$\alpha$-Amylase (1,4- $\alpha$-D-glucan-4 glucanohydrolase; EC 3.2.1.1) catalyzes the hydrolysis of the $\alpha-1,4$ glycosidic linkages of starch, thereby generating dextrins and oligosaccharides with low polymerization. ${ }^{45}$ It is universally distributed throughout the animal, plant and microbial kingdoms. ${ }^{46}$ In the genome of Caldicellulosiruptor sp. F32, five putative GH13 $\alpha$-amylases were predicted. Among them, a family 13 glycoside hydrolase, located at 329573 to 330500 (reverse), contains an N-terminal signal peptide, whereas the other four do not have. Two GH13 $\alpha$-amylases were flanked with CBM48 and CBM34, respectively (Fig. 1). Both CBMs from family 48 and 34 are starch or glycogenbinding modules. A family 15 glycoside hydrolase, predicted as a secreted protein, was annotated as glucoamylase (EC 3.2.1.3), which is exo-acting amylase that releases glucose from the nonreducing end of starch and related oligosaccharides. ${ }^{47}$ Pullulanase (EC 3.2.1.41) is a de-branching enzyme that cleaves the 1,6- $\alpha$-glucosidic linkages in pullulan or other amylaceous polysaccharides. ${ }^{48}$ In the genome of Caldicellulosiruptor sp. F32, two GH13 putative pullulanases were predicted. One contains the GH13 catalytic domain, a signal peptide, an N-terminal CBM41 and a C-terminal CBM2. The other GH13 putative pullulanase is also a secreted protein flanked by an N-terminal CBM48 (Fig. 1). Altogether, 8 starch-degrading enzymes consist of a complex CAZyme system for pullulan and starch degradation.

\section{$\beta-1,3-1,4-G l u c a n$ degradation}

$\beta-1,3-1,4-G l u c a n$ (lichenin), a liner polysaccharide containing up to $1200 \beta$-D-glucosyl residues, is an important structural and storage polysaccharide in the endosperm cell walls of cereals

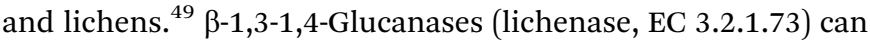
selectively hydrolyze $\beta-1,4$ glycosidic bonds in 3-O-substituted glucosyl residues. ${ }^{49}$ A GH5 enzyme F32EG5 (GenBank accession number KC958503, Fig. 1) showed the highest amino acid identity of 53\% with an endoglucanase CelCCA (YP_002505438) from $C$. cellulolyticum and was proved to be a lichenase in Caldicellulosiruptor sp. F32 (data unpublished). The analysis of hydrolysis products indicated that F32EG5 cleaved the $\beta-1,4$ linkage while the 4 - $O$-substitued glucose residue linked to a 3-O-substited glucose residue, as well as the $\beta-1,3$ linkage, which is completely different from extensively studied GH16 lichenase, which catalyzes strict endo-hydrolysis of the $\beta$-1,4-glycosidic linkage adjacent to a 3-O-substitued glucose residue in the mixed linked $\beta$-glucans, ${ }^{49}$ suggesting that F32EG5 is a novel type lichenase with specific substrate specificity and glycosidic bond cleavage patterns. A family 16 glycoside hydrolase, consisting of a signal peptide, two N-terminal SLH domains, a GH 16 catalytic domain, and six C-terminal CBMs with predicted endo- $\beta-1,3-$ glucanase (EC 3.2.1.39) activity, was found in the genome of strain F32 (Fig. 1). endo- $\beta-1,3$-Glucanases are hydrolase specific to $O$-glycoside bonds between 1,3-linked glucopyranose residues found in variety of $\beta-1,3$-glucan including laminarin, yeast $\beta$-1,3-1,6-glucan, and $\beta$-1,3-1,4-glucan. ${ }^{50}$

\section{Other possible lignocellulose-degrading related modules}

In the genome of Caldicellulosiruptor sp. F32, there are 19 extracellular solute-binding proteins. Two dimensional electrophoresis experiments have shown that there are 23 extracellular proteins which were significantly upregulated on microcrystalline cellulose (Avicel PH-101) than glucose. ${ }^{51}$ Among them, 4 proteins are extracellular solute-binding proteins, indicating the important 
role in cellulose degradation. Yokoyama et al. have reported that multidomain enzymes of genus Caldicellulosiruptor might be recruited to regions bound by noncatalytic plant cell wallbinding proteins (PWBPs) on the plant cell wall, and thus involved in bacterial survival and lignocellulosic biomass deconstruction. ${ }^{52}$ Surface layer homology (SLH) domain protein is a distinguishing feature of genus Caldicellulosiruptor and may play a role in lignocellulose deconstruction. ${ }^{29}$ The GH5 glucanase (Csac_0678) from C. saccharolyticus DSM8903 was investigated in detail. The SLH domain did not show any impact on Csac_0678 $\mathrm{GH}$ activity, but contributed to the binding to the S-layer of cells. $^{29}$ In the genome of Caldicellulosiruptor sp. F32, $7 \mathrm{SLH}$ domain containing ORFs including two CAZymes with GH5 and GH16 catalytic domain were annotated, respectively. SLH domain-containing protein is likely to anchor the catalytic domain near cells in order to quickly absorb the oligosaccharides produced by extracellular CAZymes.

\section{Thermostable $\beta$-glucosidase BlgA synergistically facilitates enzymatic degradation of Avicel by endo-1,4- $\beta$-glucanase CelB}

Recombinant $\beta$-glucosidase BlgA contains one catalytic domain, which belongs to the GH family 1 . CelB is constituted of an N-terminal GH5 catalytic domain annotated as endoglucanase, three C-terminal SLH domains, and a family 28 CBM between GH5 and SLH domains. Further analysis indicated that CelB has an N-terminal signal peptide at residues 1-28, suggesting that it is a secreted enzyme. Orthologs of BlgA and CelB also exist in all genome sequences of eight Caldicellulosiruptor species, indicating the important role of these two enzymes in cellulose deconstruction.

These two thermostable cellulose-degrading enzymes were heterologously expressed in E. coli BL21 (DE3) and purified by His-tag mediated affinity chromatography to near-homogenous, respectively. BlgA exhibited the highest specific activity against $p$ NPC (22.1 $\left.\mathrm{U} \mathrm{mg}^{-1}\right)$ and cellobiose $\left(20 \mathrm{U} \mathrm{mg}^{-1}\right)$, indicating that it is a $\beta$-glucosidase. CelB showed hydrolysis activity with CMC (17.1 $\mathrm{U} \mathrm{mg}^{-1}$ ) and $p$ NPC (10.4 $\mathrm{U} \mathrm{mg}^{-1}$ ) as substrates (Table 3). Both of them have a temperature optimum of $75{ }^{\circ} \mathrm{C}$ and a $\mathrm{pH}$ optimum of 5.6. The half-lives of recombinant BlgA and CelB at $75{ }^{\circ} \mathrm{C}$ were $48 \mathrm{~h}$ and $34 \mathrm{~h}$, respectively.

Various cello-oligosaccharides (C2-C6) were incubated with BlgA, after 12 hours, all substrates were converted to glucose completely (Fig. 2A). It has been reported that glucose tolerant $\beta$-glucosidase has significant advantage in the process of saccharification of lignocellulosic materials. ${ }^{53}$ The search for $\beta$-glucosidases insensitive to high concentration of glucose has received a lot of attention, but few of them focus on thermostable enzyme. In this study, the specific activity of $\beta$-glucosidase BlgA was gradually inhibited while increasing the concentration of glucose, and a $K_{\mathrm{i}}$ of $350 \mathrm{mM}$ glucose for BlgA was determined, which is higher than the $K_{\mathrm{i}}$ value of $200 \mathrm{mM}$ reported for $\beta$-glucosidase from Scytalidium thermophilum $^{54}$ (Fig. 2B). The $K_{\mathrm{i}}$ value of other $\beta$-glucosidase from Aspergillus niger, Thermoanaerobacterium thermosaccharolyticum, and A. tubingensis has been reported to be 543600 , and $600 \mathrm{mM}$, respectively, which indicated that these enzymes are highly glucose-tolerant. ${ }^{55-57}$ BlgA can efficiently eliminate the productinhibitors of endoglucanases during lignocellulosic biomass hydrolysis.

The degradation capacity of CelB was investigated in a time course approach with Avicel as a substrate (Fig. 2C), and most of the released products were determined to be glucose and cellobiose, which is consistent with the substrate specificity results that the endoglucanase CelB exhibited high activity for $p$ NPCase. In the following experiment, in order to eliminate feedback inhibition of hydrolysis products for CelB, BlgA was added into the reaction mixture. The synergistic effect of these two thermostable enzymes was investigated. After $36 \mathrm{~h}$ incubation, the hydrolysis ability of CelB toward Avicel was improved by $65 \%, 110 \%$ and $154 \%$ in the presence of purified BlgA at the ratio of $1: 100,1: 20$ and $1: 5$ (BlgA : CelB), respectively (Fig. 2D). BlgA is boosting cellulose-degrading ability of endo-glucanase CelB with the degree of synergy of 2.54 at the ratio of $1: 5$ (BlgA:CelB). Apparently, thermostable $\beta$-glucosidase BlgA synergistically facilitated enzymatic degradation of Avicel by CelB via removing cellobiose which inhibits the enzyme activity. Furthermore, possible accessible substrate subsites may be provided to BlgA during the hydrolysis of Avicel by CelB, which enhanced the synchronous action of synergism. ${ }^{58}$

Caldicellulosiruptor spp. can efficiently degrade lignocellulosic biomass without pretreatment that was facilitated by a diverse set of CAZymes. Unpretreated plant biomass, such as poplar, napier, and Bermuda grasses, can be utilized by $C$. becsii. ${ }^{59}$ When unpretreated wheat straw was used as a sole carbon source, strain F32 showed better growth than DSM 8903. The secretome of strain F32 showed $0.17 \mathrm{U} \mathrm{mg}^{-1}$ of FPA, $0.47 \mathrm{U} \mathrm{mg}^{-1}$ of endoglucanase, and $8.7 \mathrm{U} \mathrm{mg}^{-1}$ of xylanase, which were higher than those of C. saccharolyticus. ${ }^{8}$ The physiological and biochemical features of strain F32 indicated that a wealthy of CAZymes including cellulase and hemicellulase, and the plus synergism effect of these enzymes, such as BlgA and CelB, jointly enabled the strain to deconstruct the complex plant cell wall.

Table 3 Enzymatic properties of BlgA and CelB

\begin{tabular}{|c|c|c|c|c|c|c|c|c|c|}
\hline Protein & $\begin{array}{l}\text { Molecular } \\
\text { weight (kDa) }\end{array}$ & Theoretical pI & $\begin{array}{l}\text { Optimal } \\
\text { temp. }\left({ }^{\circ} \mathrm{C}\right)\end{array}$ & Optimal pH & \multicolumn{4}{|c|}{ Specific activity $^{a}$} & $t_{1 / 2}^{b}(\mathrm{~h})$ \\
\hline $\lg A$ & 53.4 & 5.4 & 75 & 5.6 & 0 & 0 & $22.1 \pm 0.3$ & $20 \pm 0.03$ & 48 \\
\hline
\end{tabular}

${ }^{a}$ The experiments were performed in triplicate, and data are reported as means \pm S.D. ${ }^{b} t_{1 / 2}$, half-life of enzyme at optimal temperature. - , not detected. 
A

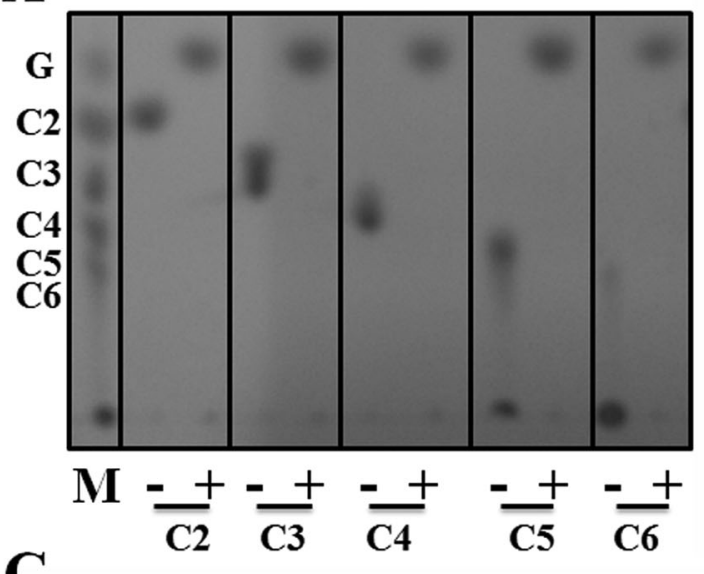

C

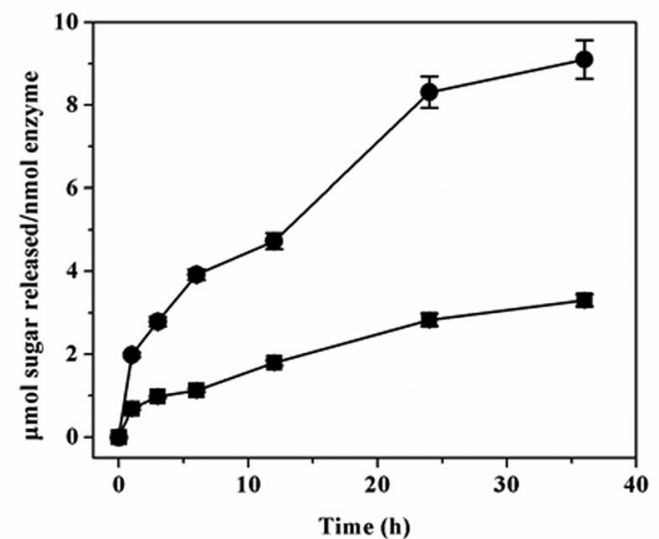

B
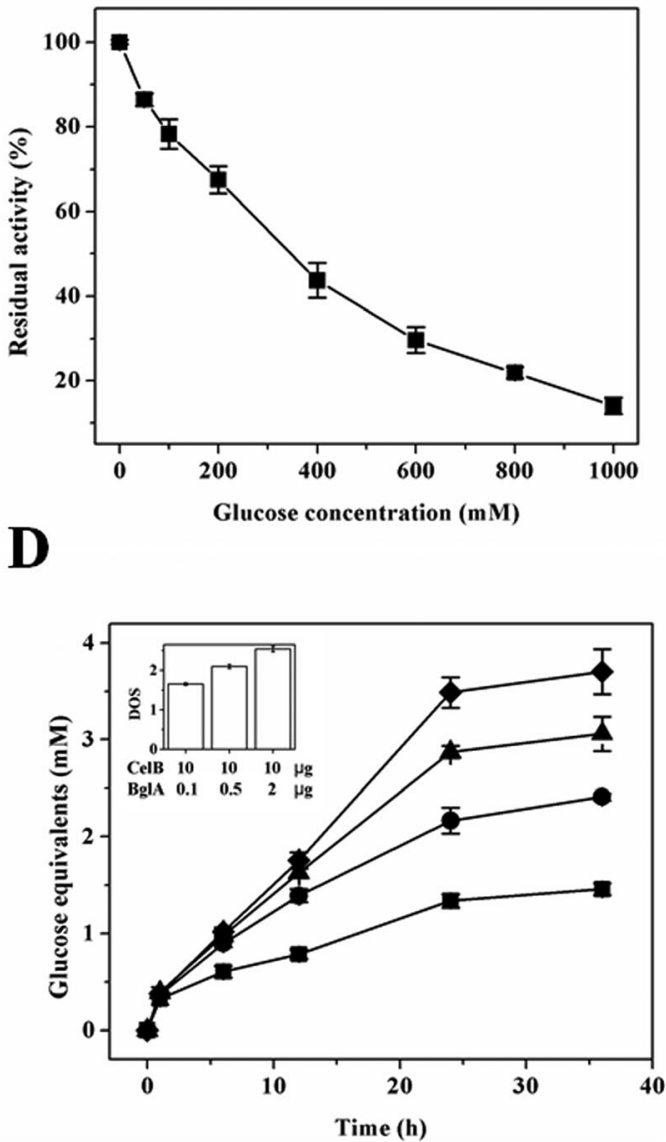

Fig. 2 Enzymatic characteristics of BlgA and CelB from Caldicellulosiruptor sp. F32. (A) Hydrolytic activity of BlgA against $\beta$-1,4-linked cellooligosaccharides. G, glucose; C2, cellobiose; C3, cellotriose; C4, cellotetraose; C5, cellopentaose; C6, cellohexaose. (B) The effects of glucose on BlgA activity. (C) Time course analysis of Avicel-hydrolysis products of CelB. Glucose, square; cellobiose, circle. (D) The changes in reducing sugar production from Avicel by synergy of CelB and BlgA. Time course analysis of Avicel hydrolysis by $10 \mu \mathrm{g}$ CelB alone (square), or synergy with $0.1 \mu \mathrm{g}$ (circle), $0.5 \mu \mathrm{g}$ (triangle), and $2 \mu \mathrm{g}$ (diamond) BlgA, respectively. The degree of synergy (DOS) of BlgA and CelB at various ratios at $36 \mathrm{~h}$ hydrolysis is shown as the inset.

\section{Conclusion}

Caldicellulosiruptor sp. F32 possesses a thermophilic CAZyme reservoir for its strong lignocellulose degradation. Most of them have convergently evolved with those from other type strains of genus Caldicellulosiruptor. Meanwhile, some CAZymes might be transferred from other organisms such as genus Clostridium via horizontal gene transfer, enabling the isolate to adapt itself to diverse environmental conditions. The thermostable $\beta$-glucosidase BlgA and endo-glucanase synergistically hydrolyze cellulose, thereby eliminating feedback inhibition of the intermediate product. All the strategies adopted by Caldicellulosiruptor sp. F32 revealed how the microbe evolved an efficient system for the utilization of carbohydrates. These results provide a basis for further development of improved enzyme mixtures aiming at industrial processing of plant biomass.

\section{Acknowledgements}

This work was supported by grants from the National Basic Research Program of China (No. 2011CB707404), the National
Science Foundation of China (No. 31400060), and the Shandong Province Natural Science Funds for Distinguished Young Scholar (JQ201507).

\section{References}

1 S. Q. Ji, D. D. Meng, K. D. Zhang and F. L. Li, in Thermophilic microorganisms, ed. F. L. Li, Caister Academic Press, Norfolk, 2015, ch. 2, pp. 13-46.

2 S. E. Blumer-Schuette, R. J. Giannone, J. V. Zurawski, I. Ozdemir, Q. Ma, Y. B. Yin, Y. Xu, I. Kataeva, F. L. Poole, M. W. W. Adams, S. D. Hamilton-Brehm, J. G. Elkins, F. W. Larimer, M. L. Land, L. J. Hauser, R. W. Cottingham, R. L. Hettich and R. M. Kelly, J. Bacteriol., 2012, 194, 4015-4028.

3 H. J. G. van de Werken, M. R. A. Verhaart, A. L. VanFossen, K. Willquist, D. L. Lewis, J. D. Nichols, H. P. Goorissen, E. F. Mongodin, K. E. Nelson, E. W. J. van Niel, A. J. M. Stams, D. E. Ward, W. M. de Vos, J. van der Oost, R. M. Kelly and S. W. M. Kengen, Appl. Environ. Microbiol., 2008, 74, 6720-6729. 
4 A. L. VanFossen, M. R. A. Verhaart, S. M. W. Kengen and R. M. Kelly, Appl. Environ. Microbiol., 2009, 75, 7718-7724.

5 R. Brunecky, M. Alahuhta, Q. Xu, B. S. Donohoe, M. F. Crowley, I. A. Kataeva, S. J. Yang, M. G. Resch, M. W. Adams, V. V. Lunin, M. E. Himmel and Y. J. Bomble, Science, 2013, 342, 1513-1516.

6 S. E. Blumer-Schuette, S. D. Brown, K. B. Sander, E. A. Bayer, I. Kataeva, J. V. Zurawski, J. M. Conway, M. W. W. Adams and R. M. Kelly, FEMS Microbiol. Rev., 2014, 38, 393-448.

7 Y. Ying, D. D. Meng, X. H. Chen and F. L. Li, Enzyme Microb. Technol., 2013, 53, 194-199.

8 Y. Ying, D. D. Meng and F. L. Li, Microbiology China, 2014, 41, 211-217.

9 D. D. Meng, Y. Ying, X. H. Chen, M. Lu, K. Ning, L. S. Wang and F. L. Li, Appl. Environ. Microbiol., 2015, 81, 2006-2014.

10 L. V. Andreou, Methods Enzymol., 2013, 529, 143-151.

11 A. L. Delcher, K. A. Bratke, E. C. Powers and S. L. Salzberg, Bioinformatics, 2007, 23, 673-679.

12 J. Besemer and M. Borodovsky, Nucleic Acids Res., 2005, 33, W451-W454.

13 F. B. Guo, H. Y. Ou and C. T. Zhang, Nucleic Acids Res., 2003, 31, 1780-1789.

14 D. A. Benson, I. Karsch-Mizrachi, D. J. Lipman, J. Ostell and E. W. Sayers, Nucleic Acids Res., 2011, 39, D32-D37.

15 M. Kanehisa, S. Goto, Y. Sato, M. Furumichi and M. Tanabe, Nucleic Acids Res., 2012, 40, D109-D114.

16 Y. B. Yin, X. Z. Mao, J. C. Yang, X. Chen, F. L. Mao and Y. Xu, Nucleic Acids Res., 2012, 40, W445-W451.

17 A. Marchler-Bauer, S. N. Lu, J. B. Anderson, F. Chitsaz, M. K. Derbyshire, C. DeWeese-Scott, J. H. Fong, L. Y. Geer, R. C. Geer, N. R. Gonzales, M. Gwadz, D. I. Hurwitz, J. D. Jackson, Z. X. Ke, C. J. Lanczycki, F. Lu, G. H. Marchler, M. Mullokandov, M. V. Omelchenko, C. L. Robertson, J. S. Song, N. Thanki, R. A. Yamashita, D. C. Zhang, N. G. Zhang, C. J. Zheng and S. H. Bryant, Nucleic Acids Res., 2011, 39, D225-D229.

18 M. Punta, P. C. Coggill, R. Y. Eberhardt, J. Mistry, J. Tate, C. Boursnell, N. Pang, K. Forslund, G. Ceric, J. Clements, A. Heger, L. Holm, E. L. L. Sonnhammer, S. R. Eddy, A. Bateman and R. D. Finn, Nucleic Acids Res., 2012, 40, D290-D301.

19 J. D. Thompson, D. G. Higgins and T. J. Gibson, Nucleic Acids Res., 1994, 22, 4673-4680.

20 E. Gasteiger, A. Gattiker, C. Hoogland, I. Ivanyi, R. D. Appel and A. Bairoch, Nucleic Acids Res., 2003, 31, 3784-3788.

21 T. N. Petersen, S. Brunak, G. von Heijne and H. Nielsen, Nat. Methods, 2011, 8, 785-786.

22 M. M. Bradford, Anal. Biochem., 1976, 72, 248-254.

23 N. Hu, B. Yuan, J. Sun, S. A. Wang and F. L. Li, Appl. Microbiol. Biotechnol., 2012, 95, 1359-1368.

24 M. Iakiviak, R. I. Mackie and I. K. Cann, Appl. Environ. Microbiol., 2011, 77, 7541-7550.

25 A. Levasseur, E. Drula, V. Lombard, P. M. Coutinho and B. Henrissat, Biotechnol. Biofuels, 2013, 6, 41.

26 C. Herve, A. Rogowski, A. W. Blake, S. E. Marcus, H. J. Gilbert and J. P. Knox, Proc. Natl. Acad. Sci. U. S. A., 2010, 107, 15293-15298.
27 A. Lochner, R. J. Giannone, M. Rodriguez, M. B. Shah, J. R. Mielenz, M. Keller, G. Antranikian, D. E. Graham and R. L. Hettich, Appl. Environ. Microbiol., 2011, 77, 4042-4054.

28 Z. L. Yi, X. Y. Su, V. Revindran, R. I. Mackie and I. Cann, PLoS One, 2013, 8, e84172.

29 I. Ozdemir, S. E. Blumer-Schuette and R. M. Kelly, Appl. Environ. Microbiol., 2012, 78, 768-777.

30 B. C. Saha, J. Ind. Microbiol. Biotechnol., 2003, 30, 279-291.

31 F. M. Girio, C. Fonseca, F. Carvalheiro, L. C. Duarte, S. Marques and R. Bogel-Lukasik, Bioresour. Technol., 2010, 101, 4775-4800.

32 T. Collins, C. Gerday and G. Feller, FEMS Microbiol. Rev., 2005, 29, 3-23.

33 K. Zhang, X. Chen, W. H. Schwarz and F. Li, Appl. Environ. Microbiol., 2014, 80, 2592-2601.

34 A. L. Van Fossen, I. Ozdemir, S. L. Zelin and R. M. Kelly, Biotechnol. Bioeng., 2011, 108, 1559-1569.

35 D. Guillen, S. Sanchez and R. Rodriguez-Sanoja, Appl. Microbiol. Biotechnol., 2010, 85, 1241-1249.

36 A. B. Boraston, D. N. Bolam, H. J. Gilbert and G. J. Davies, Biochem. J., 2004, 382, 769-781.

37 J. Rangasamy, N. Nitar, N. Hideaki, F. Tetsuya and T. Hiroshi, Macromol. Symp., 2008, 264, 163-167.

38 D. Bhattacharya, A. Nagpure and R. K. Gupta, Crit. Rev. Biotechnol., 2007, 27, 21-28.

39 A. S. Sahai and M. S. Manocha, FEMS Microbiol. Rev., 1993, 11, 317-338.

40 H. Shibasaki, K. Uchimura, T. Miura, T. Kobayashi, R. Usami and K. Horikoshi, Appl. Microbiol. Biotechnol, 2014, 98, 7845-7853.

41 A. Durand, R. Hughes, A. Roussel, R. Flatman, B. Henrissat and N. Juge, FEBS J., 2005, 272, 1745-1755.

42 B. L. Ridley, M. A. O'Neill and D. A. Mohnen, Phytochemistry, 2001, 57, 929-967.

43 L. M. Blackman, D. P. Cullerne and A. R. Hardham, BMC Genomics, 2014, 15, 785.

44 P. Dam, I. Kataeva, S. J. Yang, F. F. Zhou, Y. B. Yin, W. C. Chou, F. L. Poole, J. Westpheling, R. Hettich, R. Giannone, D. L. Lewis, R. Kelly, H. J. Gilbert, B. Henrissat, Y. Xu and M. W. W. Adams, Nucleic Acids Res., 2011, 39, 3240-3254.

45 R. Rodriguez-Sanoja, B. Ruiz, J. P. Guyot and S. Sanchez, Appl. Environ. Microbiol., 2005, 71, 297-302.

46 R. Gupta, P. Gigras, H. Mohapatra, V. K. Goswami and B. Chauhan, Process Biochem., 2003, 38, 1599-1616.

47 S. K. Gudi, C. Gurramkonda, G. Rather, M. G. S. Chandra, U. K. Mangamuri, S. Podha and Y. L. Choi, J. Korean Soc. Appl. Biol. Chem., 2013, 56, 427-433.

48 R. S. Singh, G. K. Saini and J. F. Kennedy, Carbohydr. Polym., 2011, 83, 672-675.

49 N. Planas, Biochim. Biophys. Acta, 2000, 1543, 361-382.

50 B. W. Zhu, J. G. Zhao, J. F. Yang, T. Mikiro, Z. S. Zhang and D. Y. Zhou, Process Biochem., 2008, 43, 1102-1106.

51 Y. Ying, PhD thesis, University of Chinese Academy of Sciences, 2012.

52 H. Yokoyama, T. Yamashita, R. Morioka and H. Ohmori, J. Bacteriol., 2014, 196, 3784-3792. 
53 J. J. Pei, Q. Pang, L. G. Zhao, S. Fan and H. Shi, Biotechnol. Biofuels, 2012, 5, 31.

54 F. F. Zanoelo, L. Polizeli Mde, H. F. Terenzi and J. A. Jorge, FEMS Microbiol. Lett., 2004, 240, 137-143.

55 T. R. Yan and C. L. Lin, Biosci., Biotechnol., Biochem., 1997, 61, 965-970.

56 C. H. Decker, J. Visser and P. Schreier, Appl. Microbiol. Biotechnol., 2001, 55, 157-163.
57 J. Pei, Q. Pang, L. Zhao, S. Fan and H. Shi, Biotechnol. Biofuels, 2012, 5, 31.

58 K. Riedel, J. Ritter and K. Bronnenmeier, FEMS Microbiol. Lett., 1997, 147, 239-243.

59 S. J. Yang, I. Kataeva, S. D. Hamilton-Brehm, N. L. Engle, T. J. Tschaplinski, C. Doeppke, M. Davis, J. Westpheling and M. W. Adams, Appl. Environ. Microbiol., 2009, 75, 4762-4769. 